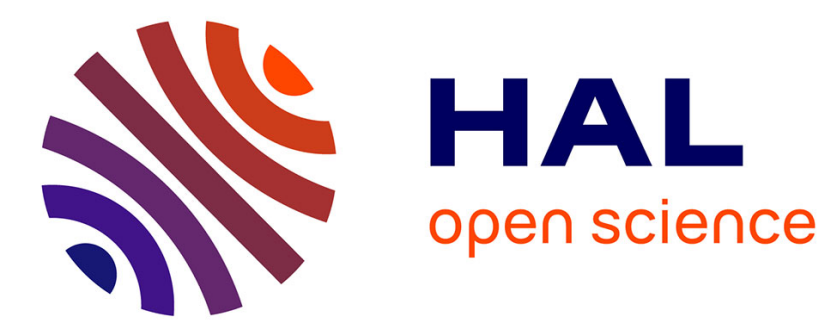

\title{
De la route-monument au réseau routier
}

Sandrine Robert

\section{To cite this version:}

Sandrine Robert. De la route-monument au réseau routier. Les Nouvelles de l'archéologie, 2009, 115, pp.8-12. 10.4000/nda.631 . halshs-00442787

\section{HAL Id: halshs-00442787 https://shs.hal.science/halshs-00442787}

Submitted on 22 Dec 2009

HAL is a multi-disciplinary open access archive for the deposit and dissemination of scientific research documents, whether they are published or not. The documents may come from teaching and research institutions in France or abroad, or from public or private research centers.
L'archive ouverte pluridisciplinaire HAL, est destinée au dépôt et à la diffusion de documents scientifiques de niveau recherche, publiés ou non, émanant des établissements d'enseignement et de recherche français ou étrangers, des laboratoires publics ou privés. 


\title{
De la route-monument au réseau routier
}

\author{
S. Robert, \\ Maître de conférence associé Université de Paris I, UMR 7041 Arscan Nanterre et Conseil général du Val-d’Oise
}

\section{Résumé}

La recherche sur le réseau routier antique, théorisée très tôt grâce à l'existence de sources littéraires et épigraphiques, a privilégié en archéologie une approche monumentale de la route au détriment du réseau. Ces dernières années, les études archéogéographiques ont montré le réseau routier comme un faisceau de tracés mobiles à moyenne et grande échelle mais présentant une certaine pérennité à l'échelle des itinéraires régionaux. Le trafic routier s'appuie sur une structure matérielle complexe, assurant la résilience du système à petite échelle. En croisant la modélisation des réseaux et la reconstitution de leur réalité matérielle, on peut mieux comprendre leur dynamique dans le temps à travers la concurrence entre les pôles.

Mots-clés : réseau routier, histoire de la recherche, archéogéographie, resilience, échelle

\begin{abstract}
Antique roads research has been theorised earlier because of the presence of texts and epigraphy. It has prioritized monumental vision of road to the detriment of network perception. Over the last years, archaeogeographs have revealed that, if road sections move at local scale, itinerary remains permanent at regional scale. On a small scale, a complex structure guarantees the resilience of the system. Intersecting network modelling and structure reconstruction, we can understand their dynamics over a long period of time. It is based on competition between centres.
\end{abstract}

Keywords : road network, history of research, archaeogeography, resilience, scale

\section{La route comme «monument »}

Les voies anciennes ont été traitées dès la Renaissance comme un chapitre obligé des travaux de topographie historique (Chevallier 2000 : 19). Elles apparaissaient couramment dans les monographies régionales réalisées par des érudits aux XVIIe et XVIIIème siècles ${ }^{1}$ et le XIXe siècle, avec les sociétés savantes naissantes, est une période très féconde dans la publication d'articles et de synthèses régionales ou départementales sur les routes antiques. Les savants utilisaient les témoignages des agents voyers et les relevés topographiques exécutés à partir de 1817 pour la confection de la carte d'Etat-major ${ }^{2}$. En 1858, un recensement des itinéraires et des bornes milliaires est entrepris par la Commission de topographie des Gaules, instituée par Napoléon III (Bertrand 1864).

Parrallèlement à ces démarches d'inventaire, apparaît très tôt une théorie et une méthode d'étude des voies. En effet, dès 1622, N. Bergier, propose, dans son Histoire des grands chemins de l'Empire romain, une théorie sur la structure des voies romaines à partir des textes et des observations de terrain. Á sa suite, les fondateurs de l'archéologie gallo-romaine en France consacrèrent systématiquement une partie de leurs manuels à ce sujet - Daremberg et Saglio dans leur Dictionnaire des antiquités ... écrit entre 1877 et 1906 (Daremberg et Saglio 1969 : 784-786), C. Jullian dans son Histoire de la Gaule écrite entre 1907 et 1921 (Jullian 1920 : 81-132), A. Grenier dans le tome II de son Manuel d'archéologie gallo-romaine

1 Par exemple, dans son Recueil d'Antiquités, paru entre 1752 et 1767, le Comte de Caylus décrit un grand nombre de voies romaines à partir d'observations directes et minutieuses sur le terrain. Il s'appuyait notamment sur des relevés envoyés par les ingénieurs des Ponts et Chaussées (Schnapp 1993 : 307).

2 Les officiers du Dépôt de la guerre eux-mêmes recueillent à l'occasion de leurs opérations de terrain, des témoignages et des observations sur ces anciens axes. Ils sont consignés dans leurs « Mémoires et

Reconnaissances » conservés au Service Historique de la Défense. 
consacré aux routes publié en 1934 (Grenier 1985), etc.

Dans ces ouvrages, l'origine des réflexions se situe dans les textes classiques. L'accent est mis sur les routes d'origines prestigieuses, citées dans les sources, puis sur les vestiges monumentaux les plus apparents : bornes milliaires monumentales, stations routières, ponts, superstructures. La terminologie et le classement des voies sont déduits essentiellement des textes ou des inscriptions sur les bornes milliaires, quitte parfois à adapter un vocabulaire qui n'est pas directement dédié aux routes. En effet, les textes de Vitruve et de Pline l'Ancien cités par $\mathrm{N}$. Bergier et ses successeurs se rapportent en réalité à la construction des sols dans les habitations. Jouant sur un faux syllogisme entre les termes pavimentum et pavé, N. Bergier transfère ce vocabulaire à la description des routes proposant alors une typologie alternant une succession de couches construites -statumen, rudus ou ruderatio, nucleus, summum dorsum(Chevallier 1972 : 94). Mais cette typologie se révèle peu applicable sur le terrain comme le souligneront Bergier lui-même et les érudits des siècles suivants (Grenier 1985 : 321-325). Par ailleurs, cette typologie ne s'adapte qu'à la description des voies construites en dur et laisse dans l'ombre les voies moins élaborées qui peuvent pourtant être importantes dans la hiérarchie fonctionnelle du réseau. Cette typologie a contribué à faire de la voie construite, un objet monumental que l'on a traditionnellement rapporté à deux grandes périodes de constructions routières en Gaule : une qui correspondrait au réseau d'Agrippa, exposé par Strabon à la fin du règne d'Auguste, et une autre attribuée au règne de Claude, empereur souvent cité sur les bornes milliaires (Grenier 1985: 42-51). L'attribution de ces constructions à des faits historiques marquants (conquête de la Gaule et campagne de Bretagne) renforçait encore leur monumentalité. En réalité, les datations recueillies en fouille stratigraphique sont très diverses et elles nous renseignent souvent plus sur l'abandon de la voie par le comblement des fossés bordiers que sur sa construction.

Le manuel qui fait toujours référence sur les voies romaines est publié en 1972 par R. Chevallier et réédité en 1998 (Chevallier 1972). Il s’appuie sur les recherches précédemment citées $^{3}$. Les renvois aux sources historiques et épigraphiques sont prépondérants dans la méthode d'étude qu'il propose mais il développe aussi l'analyse de cartes topographiques et de photographies aériennes qui, dès les années 1970, permettent de recomposer les grandes voies disparues à travers la succession d'un chemin, d'une route goudronnée ou d'une simple haie. Mais, la prospection aérienne met l'accent encore sur les tracés monumentaux rectilignes, plus facilement repérables du ciel. Cette caractéristique était indiquée dès le XIXe siècle comme une particularité distincte des voies romaines et associées selon A.Grenier aux " grandes voies d’intérêt général » (Daremberg et Saglio 1969 : 786, Grenier 1985 : 178-180).

Dans cette recherche, on confond souvent itinéraire et tracé. L'exemple le plus flagrant est l'utilisation qui a été faite jusqu'à maintenant de la Table de Peutinger, considérée souvent comme une carte topographique représentant le réseau physique alors qu'il s'agit plus probablement d'un graphe (série de pôles reliés par des traits théoriques symbolisant une relation $)^{4}$. P. Herrmann montre d'ailleurs que les mentions de distances sur la Table peuvent indiquer au mieux des distances à vol d'oiseau mais en aucun cas des mesures de tracés réels (Herrmann 2007). Or, trop souvent on essaie encore d'associer un tracé unique et prestigieux à un arc de la " carte » alors qu'à un itinéraire donnée peuvent correspondre plusieurs tracés potentiels, ce qui explique les nombreuses controverses nées depuis le XIXème siècle. En effet, on tente de faire coïncider les découvertes de terrains ou des lieux particuliers (civitates, stations routières, sanctuaires etc.) avec ce qui s’apparente à des itinéraires plus qu'à une

3 Il s’inspire notamment du manuel d'A. Grenier auquel il dédie son livre.

$4 \mathrm{Ph}$. Mathis propose d'y voir d'ailleurs une des représentations fonctionnelles les plus anciennes d'un réseau routier : un graphe associant des pôles (les étapes), des arcs (les traits représentés) et des valuations de ces arcs (les indications de distance) cf. Mathis 2003 : 38-39 
cartographie topographique. La complémentarité entre réseau fluvial et routier est peu abordée et la relation route-rivière est souvent perçue uniquement par la question du franchissement car elle permet de mettre en valeur des ouvrages monumentaux.

Si l'on considère le réseau de circulation avant tout dans sa dimension physique et monumentale, la reconstitution ne peut passer alors que par la cartographie de la somme des tronçons mis au jour dans des fenêtres archéologiques ou attestés par des sources historiques. Or, cette méthode se confronte rapidement à une discontinuité des sources puisque la reconstitution dépend des conditions de conservation des vestiges anciens. Cette difficulté est augmentée à proportion de l'espace couvert par l’étude.

Figure 1 :

En France, les manuels d'étude des voies anciennes publiés par A. Grenier en 1934 (réédité en 1985 ) et R. Chevallier en 1972 (réédité en 1998) privilégient l'étude des voies monumentales.

\section{La route « faisceau »}

Dans les années 1990, un saut conceptuel est franchi lorsque la recherche commence à porter sur l'ensemble du réseau plutôt que sur des axes pré-déterminés par l'analyse des sources antiques. Dans le pays de Vaud, E. Vion propose de raisonner sur l'ensemble du réseau observable sur les plans contemporains plutôt que sur la somme de tronçons reconnus par la fouille ou les sources historiques (Vion 1989 : 69). Pour la première fois, l'analyse traite le réseau actuel comme un ensemble et ne se contente plus de tenter d'y discerner quelques axes remarquables. Des tris graphiques (cf. C. Marchand infra.) ont permis de dégager des faisceaux de voies complexes s'organisant dans des itinéraires. L'intérêt de cette démarche est de donner une localisation géographique précise des tronçons et de pouvoir observer la complexité du réseau dans le temps. Sur ce modèle, plusieurs études ont été réalisées dans diverses régions de France (Marchand 1997, Leturcq 1997, Jung 1999).

Ces études archéogéographiques ont montré que pour un itinéraire donné, plusieurs tracés peuvent se succéder voire coexister. Dans le canton de Vaud, E. Vion signale dès 1989, ces innombrables «branches » entrant dans un itinéraire donné. Il propose de leur donner une origine pré-romaine (Vion 1989: 90). Dans le Tricastin, C. Jung a montré que ce fonctionnement en faisceau pouvait exister dès la période antique avec des tracés constituant des diverticules de voies plus importantes comme la via d'Agrippa (Jung 1999 : 92-119). Dans le Val-d'Oise, les dix itinéraires que nous avons étudiés associent plusieurs tracés et seuls quatre itinéraires comportent un tracé monumental rectiligne. Les autres sont relativement sinueux et semblent nés de l'usage plus que d'un programme spécifique de construction. Ils présentent cependant une solution de continuité à l'échelle régionale. Les observations faites en fouille ont livré des datations du Ier siècle au IIIe siècle ap. J. C. pour les voies monumentales et du Ier ap ; J. C. à la période carolingienne pour les autres tracés (cf. $\mathrm{S}$. Robert infra). Ces derniers présentaient des types de constructions très divers : simple chemin de terre, utilisation du sol géologique calcaire entayé directement par des ornières etc. Certains établissements majeurs, comme le sanctuaire antique de Genainville ou l'agglomération protohistorique et antique d'Epiais-Rhus, sont situées à proximité immédiate de ces tracés alors qu'ils sont à plusieurs kilomètres des grands axes rectilignes monumentaux connus. Les tracés non monumentaux semblent jouer un rôle également dans la localisation des nécropoles du haut Moyen-Age (Robert 2006a et b). Pour l'itinéraire Paris-Rouen, P. Ouzoulias a déduit le même type de fonctionnement en faisceau à partir de l'étude des registres des visites paroissiales effectuées par Eudes Rigaud, archevêque de Rouen, entre 1248 et 1275. Dans le Vexin, hormis la grande voie antique dite Chaussée Jules-César, 
l'archevêque utilisait deux autres tracés dont un Vieux Chemin, utilisé préférentiellement vraisemblablement parcequ'il desservait certaines des paroisses à visiter. Il s'agissait alors ici d'une « spécialisation » selon un but donné (Ouzoulias 1991).

En Beauce, S. Leturcq a étudié des tracés multiples entre Etampes et la forêt d'Orléans. Au moins deux branches principales parrallèles Paris-Orléans semblent coexister au haut MoyenAge. Elles relèvent selon lui d'usages différents : la branche orientale constituant une liaison rapide pour les voyageurs légers, la branche médiane (actuelle RN 20) étant la route commerciale empruntée par les pondéreux (Leturcq 1997 : 84-85).

Dans le Senonnais, C. Marchand montre l'existence de ce type de faisceau et propose un développement antérieur au XIIe siècle - peut-être contemporain du VIIe siècle dans le casde l'itinéraire Montereau-Fault-Yonne - Nemours (Marchand 1997 et infra).

B. Lepetit qui étudie le réseau routier du XVIIIe au XIXe siècle à travers les enquêtes administratives a montré au début des années 1980 cette incessante mobilité des tracés précisant que la route comme support matériel du flux que tentent de capter les pouvoirs publics "compte moins que les orientations des mouvements et la scansion régulière des relais " (Lepetit 1984: 19). Il semble que cette mobilité des tracés bien attestée par les sources modernes, soit courante aussi dans les périodes anciennes. D’ailleurs, en 1934, A. Grenier, à la suite de C. Jullian, signalait déjà des « voies doubles » c'est-à-dire « ayant même point de départ et même direction générales qui courent, plus ou moins parallèles, à une distance variable l'une de l'autre. ». Il proposait d'y voir les vestiges de routes gauloises doublées par les routes romaines et coexistant encore ou non avec celle-ci (Grenier 1985 : 392-401).

Figure 2 : En travaillant sur le réseau transmis et en dégageant des faisceaux de tracés, E. Vion a donné une nouvelle impulsion à la recherche sur les voies en archéogéographie au début des années 1990 (Vion 1989).

\section{Un réseau résilient}

La mise en évidence de ces faisceaux complexes montre qu'il faut notamment dissocier la notion d'itinéraire et celle de tracé (Lepetit 1984, Vion 1989, Verdier 2007). On peut distinguer trois niveaux dont l'articulation produit la dynamique des réseaux routiers dans le temps. L'itinéraire correspond au flux de circulation entre deux pôles importants (villes, ponts, cols, péages, points de ruptures de charge etc.), empruntant des tronçons de voies différents coexistant ou se déplaçant dans le temps. Les études archéogéographiques montrent une grande pérennité des itinéraires de l'Antiquité, voire de la Protohistoire à aujourd'hui. Le tracé matérialise le flux dans une emprise au sol. Il associe des tronçons disparates d'origines très différentes. Les tracés conservent rarement leur fonction de "grand parcours » de l'Antiquité à aujourd'hui ${ }^{5}$. Le modelé joue alors un rôle déterminant dans cette relation dynamique entre forme, fonction et statut de la voie. Forme construite de la voie, il autorise un certain type de viabilité et de fonctionnement du tracé. Les réfections sont constantes en fonction de l'usure et de l'évolution technique des moyens de locomotion. Lors des fouilles, on perçoit bien les recharges et reprises incessantes. C'est au niveau des tracés et du modelé que se joue la résilience du réseau, dans sa capacité à transformer des formes préexistantes dans une réadaptation incessante des fonctions. De l'articulation des trois niveaux : itinéraire, tracé et modelé, nait la dynamique du réseau routier dans le temps

5 Dans le Val-d'Oise par exemple, ils gardent cette fonction rarement au-delà de trois à cinq siècles mais on note qu'un tronçon déclassé peut reprendre de l'importance après un temps de hiatus dans sa fonction (Robert à paraître). 
En effet, de façon récurente, les flux régionaux entrent en conflit avec les réalités locales : topographie contraignante, murs et portes des villes, densification du bâti, viabilité inadéquate de la voie, volontés d'appropriation des axes de circulation, concurrence des centres ... Mais, les «captures » des axes ne remettent pas forcément en cause l'itinéraire et contribuent parfois à le maintenir en assurant l'entretien de la route à des périodes où l'état central ne l'assure plus globalement. Inversement, le passage d'une voie de grand parcours est aussi un facteur de pérennité pour l'habitat local, surtout associé à une implantation topographique particulière (éperon, gué etc.) et à un carrefour routier de plusieurs itinéraires (Robert 2006a, Verdier infra). Création de nouveaux tracés mais aussi, dans de nombreux cas, réutilisation de tronçons existants permettent de maintenir l'itinéraire. L'adaptation de la forme à la fonction par le modelé, souvent avec un certain décalage, permet d'assurer la viabilité de la voie.

Le réseau hérité apparaît ainsi comme un facteur de résilience dans la mesure où il fournit un réservoir potentiel de tronçons qui peuvent être réempruntés (Robert 2003 et infra).

L'interaction entre flux, tracé et modelé produit donc une dynamique entre le local et le régional qui est facteur de résilience, chaque échelle constituant le facteur de conservation et de transformation de l'autre. L'approche archéogéographique montre qu'il est nécessaire aujourd'hui d'étudier les voies non plus comme des objets monumentaux déconnectés de leur territoire mais comme des objets appartenant à des réseaux complexes de relation, mobilisant plusieurs échelles spatiales et temporelles. L'archéologie de terrain renseigne avant tout sur le modelé des tracés. L'étude morphologique à partir des cartes anciennes et photographies aériennes permet de percevoir les tracés et de les associer dans des itinéraires. L’archéologie spatiale peut permettre d'appréhender le flux, élément constitutif du réseau, qui associe dans un itinéraire des tracés et modelés divers en fonction d’un usage donné.

\section{La modélisation du trafic routier}

Dans les années 1980, B. Lepetit signalait la difficulté à saisir la réalité matérielle du réseau routier même lorsqu'il est bien documenté par les textes comme les routes de la France moderne (Lepetit 1984). Le caractère anastomosé et fluctuant des déplacements et la nature des sources écrites et cartographiques dont on dispose engagent à raisonner sur des relations entre des points plus que sur des tracés déterminés (cf. N. Verdier infra).

Pour les périodes anciennes, la modélisation des relations supposées entre les sites archéologiques permet d'aborder le réseau de circulation. Renforcés ces dernières années par l'utilisation des Systèmes d'Information Géographique (SIG), des modèles de diffusion spatiale des artefacts tels que les modèles de gravité ont été testés par les archéologues. Ils supposent qu'il existe une concurrence spatiale entre des centres et que les relations fonctionnelles entre centres principaux et secondaires décroissent en fonction de la taille des centres et de la distance (Djijian 1991 et cf. trois expériences récentes dans Nuninger et Sanders 2006 et al.).

De la localisation spatiale des vestiges (objets ou établissements) sont déduites des relations fonctionnelles entre des centres. Se pose alors la question du réseau de transport qui unit les lieux car, pour les périodes anciennes les échanges s’opèrent essentiellement par contact physique et se cristalisent sur un réseau de circulation inscrit dans l'espace qu'il soit routier ou fluvial. Un semis de points peut être rapporté à un axe par une simple transformation cartographique linéaire : le long d'un fleuve ou d'un axe connu, par exemple (Mordant et al. 2006). Cette méthode présente l'intérêt de s'appuyer non pas sur un axe théorique mais sur un axe réel de circulation mais on utilise plus couramment la méthode du plus court chemin 
entre un ou plusieurs points sources pour modéliser un cheminement potentiel. Ces travaux ont été développés sur des territoires ayant donné lieu à de solides opérations d'inventaires et de prospections qui permettent d'avoir une bonne connaissance de la dispersion des établissements humains.

Le plus court chemin est calculé à partir d'algorithmes comme celui de Dijkstra (cf. A. Chevalier infra) en prenant en compte différentes contraintes dites de coûts : le plus souvent la pente (Garmy et al. 2005, Robert 2007), à laquelle on peut ajouter la vitesse de déplacement et l'énergie requise (Ejstrud 2005, Verhagen et al. 1999), mais aussi le coût métabolique, la masse du sujet et de la charge portée (ex. modélisation des cheminements entre les oppida du Languedoc: Zakšek et al. à paraître ou l'algorithme très complet de Pandolf, utilisé par exemple dans le Wroxeter Hinterland Project Britain : Van Leusen 2002) ${ }^{6}$. La visibilité et l'intervisibilité entre des éléments marqueurs du paysage peuvent également être prises en compte (Zakšek et al. à paraître, Llobera 2003).

Le tracé réel des axes de circulation attestés est encore peu intégré dans les modéles. Pourtant, les routes introduisent une forte anisotropie dans l'espace (Pinchemel et Pinchemel 1988 : 101). Elles relativisent les notions de distance et peuvent constituer des couloirs privilégiés de déplacement intervenant dans les relations fonctionnelles entre centres. La "rugosité » introduite dans l'espace par les lignes pourrait être mieux évaluée en s’inspirant par exemple des travaux réalisés en écologie ${ }^{7}$.

Dans ce type d'analyse, la difficulté se situe dans un deuxième temps dans la confrontation du modèle avec le terrain lorsque l'on tente " d'accrocher » la reconstitution théorique sur des tronçons attestés au sol et localisés précisément. Dans le Val-d’Oise, nous avons comparé la modélisation des tracés joignant les cités antiques des régions limitrophes et les tracés mis en évidence par l'analyse archéogéographique à partir d'une base de données constituée par l'assemblage et la vectorisation du cadastre napoléonien sur les 185 communes du département (Robert à paraître). Deux modélisations ont été proposées : l'une par le chemin à vol d'oiseau, l'autre en prenant en compte la pente (Robert 2007).

Pour Beauvais, important carrefour routier antique qui a généré des voies multiples dans le Vexin et la Plaine de France, les voies reconstituées par l'analyse correspondent dans deux cas sur quatre à l'itinéraire théorique prenant en compte la pente. Mais pour l'itinéraire Orléans-Beauvais, les deux modèles sont très différents : le chemin direct traverse le Vexin évitant Paris tandis que le chemin suivant la pente passe par la future capitale. Les deux sont représentés par une voie antique monumentale au tracé rectiligne mais la chaussée Brunehaut, passant par le Vexin, n'est plus entretenue à partir de la fin de l'Antiquité tandis que la voie Paris-Beauvais a subsisté jusqu'à aujourd'hui sous la forme de la nationale 1 . Le développement de Paris comme noyau intermédiaire semble avoir joué un rôle dans cet abandon, en capturant et redistribuant ces itinéraires.

Le croisement entre modélisation et analyse archéogéographique montre donc la complexité des tracés possibles qui se cache derrière la relation entre deux points éloignés. Elle illustre aussi la dynamique de la concurrence entre les pôles qui peut amener certains tracés à tomber en désuétude même si ils sont plus directs.

Figure 3: La modélisation du trafic, confrontée aux tracés reconstitués par l'archéogéographie, permet de mieux comprendre les logiques de concurrence entre les pôles (Robert 2007).

6 Certes ces algorithmes ont souvent été développés pour des problématiques contemporaines et demanderaient à être adaptés aux sociétés anciennes. On les utilise généralement sur des modèles numériques de terrain contemporains qui ne rendent pas totalement compte de l'état du relief aux périodes considérées mais ils constituent déjà un premier jalon pour la modélisation des réseaux.

7 Pour la prise en compte des couloirs de trafic dans la diffusion d’éléments, des applications ont été réalisés notamment dans le contexte d'émissions de polluants (par ex. Decoupigny 2003, Jeannet à paraître...). 


\section{Conclusion}

L'existence d'une théorie précocement élaborée sur la base de sources historiques a eu pour effet de mettre l'accent sur la recherche de tracés monumentaux antiques facilement repérables dans le paysage par leur axe rectiligne et sur le terrain par la succession des couches construites. Or l'étude du réseau routier comme un faisceau complexe demande de prendre en compte l'ensemble des voies qu'elles soient monumentales ou non. Par ailleurs, l'approche monumentale a conduit à privilégier certaines périodes. Une rapide analyse de la base de données « Mérimée » montre que pour les 78 voies inscrites à l’Inventaire général et aux Monuments Historiques, les périodes antique, moderne et contemporaines représente 88\% des mentions (respectivement 56\%, 24\% et 8\%), la préhistoire et le Moyen-Age, seulement 3 et $4 \%$ (cf. figure 4). Or, cela ne rend pas compte de la réalité matérielle de ces périodes (cf. S. Robert infra) mais cela conduit à écrire une histoire du réseau routier par ruptures alors que les héritages et les transmissions forment l'essence de sa construction sur la longue durée. Le passage à une vision de la route comme un réseau complexe et dynamique dans le temps nécessite donc de réévaluer l'apport des périodes pré et post-romains à la construction et à la transmission des réseaux routiers dans le temps.

Figure 4 : Les voies classées MH ou inscrites à l’Inventaire Général appartiennent pour une large à l’Antiquité et la période moderne (comparer avec Robert infra, figure p. X).

\section{Bibliographie}

BERGER, J. F., BERTONCELLO, F. , BRAEMER, F., DATVIAN, G., GAZENBECK, M. (dir.) 2005. Temps et Espaces de l'Homme en société, analyses et modèles spatiaux en archéologie. XXVe rencontres internationales d'archéologie et d'histoire d'Antibes, Antibes, Editions APDCA, 534 p.

BERTRAND, A. 1864. Voies romaines en Gaule, voies des itinéraires. Résumé du travail de la Commission de la topographie des Gaules, Extrait de la Revue archéologique. Paris, Librairie académique, 61 p.

CHEVALLIER, R. 1972. Les voies romaines. Paris, Armand Colin, 313 p.

CHEVALLIER, R 2000. Lecture du temps dans l'espace. Topologie archéologique et historique. Paris, Picard, 229 p.

CHOUQUER, G. dir. 1997. Les Formes du paysage, Tome III : L'analyse des systèmes spatiaux. Paris, Éditions Errance, 198 p.

DAREMBERG, Ch. et SAGLIO, E. 1969. Article "Via, route ou rue », in : Dictionnaire des antiquités grecques et romaines : d'après les textes et les monuments. Paris, Hachette, pp. 777-817, écrit entre 1877 et 1906, $1^{\text {ère }}$ édition 1934.

DECOUPIGNY, C. 2003. «Couloirs de trafics et émissions de polluants », in : MATHIS Ph. (dir.) 2003 : 187211

DJINDJIAN, F. 1991. Méthodes pour l'archéologie. Paris, Armand Colin, 401 p.

DURAND-DASTÈS, F., FAVORY, F., FICHES, J. L., MATHIAN, H., PUMAIN, D., RAYNAUD, C., SANDERS, L. et VAN DER LEEUW, S. 1998. Archaeomedes. Des oppida aux métropoles. Archéologues et géographes en vallée du Rhône. Paris, Anthropos, Economica, 280 p. 
EJSTRUD, B. 2005. « Cost surface analysis and ancient roads : a comparison », in : BERGER J. F. et al. (dir.) 2005 : 135-139

GARMY, P., KADDOURI, L., ROZENBLAT C. et SCHNEIDER, L . "Logiques spatiales et « systèmes de villes » en Lodévois de l'Antiquité à la période moderne », in : BERGER J. F. et al. (dir.) 2005 : 225-236.

GRENIER, A. 1985. Manuel d'archéologie gallo-romaine. Tome 2, l'archéologie du sol. Navigation-occupation du sol. Paris, Editions Picard, 468 p.

HERRMANN, P. 2007. Itinéraires des voies romaines. Paris, Editions Errance, 275 p.

JEANNET, N. à paraître. « Contributions pratiques d'une géostatistique raisonnée en environnement : méthodes et application à la cartographie régionale de la pollution par le dioxyde d'azote (NO-2) en Alsace ", in : AUBRY L. et SOULIER P. dir. à paraître : Les approches spatiales des méthodes statistiques appliquées à l'archéologie. Journée d'étude organisée par le 13 décembre 2007 à la MAE de Nanterre. A paraître dans les Cahiers transversaux de l'UMR 7041

JULLIAN, C. 1920. Histoire de la Gaule, tome V,. Paris, Hachette

JUNG, C. 1999. Morphogénèse, fonctions et évolution de la centuriation B d'Orange et essai de restitution diachronique des paléopaysages du Tricastin (Drôme-Vaucluse). Thèse pour obtenir le grade de Docteur de l'Université de Tours.Tours, Université de Tours, 366 p.

LEPETIT, B. 1984. Chemins de terre et voies d'eau, réseaux de transports et organisation de l'espace en France, 1740-1840. Paris, Editions de l'EHESS, 148 p.

LETURCQ, S. 1997. «La route et le paysage. Dynamique et stabilité des réseaux routiers beaucerons entre Etampes et la forêt d'Orléans », in : CHOUQUER G. (dir.) 1997 : 66-78

LLOBERA, M. 2003. “Estending GIS-based visual analysis: the concept of visualscapes”. International Journal of Geographical Information Science Vol. 17/1 : 25- 48

MARCHAND, C. 1997. "Réseau viaire et dessin parcellaire : étude morphologique de la région du Gâtinais oriental ». In : CHOUQUER G.. (dir.) 1997 : 66-77

MATHIS, Ph. (dir.) 2003. Graphes et réseaux, modélisation multiniveau. Paris, Lavoisier, 361 p.

MORDANT, C., SALIGNY, L. POULET-CROVISIER, N. «La circulation des objets métalliques à l'âge du Bronze entre Rhône et Saône: utilisation d'une transformation cartographique linéaire ", Mappemonde n83 (32006), en ligne sur http://mappemonde.mgm.fr

NUNINGER, L. et SANDERS, L. 2006. « La modélisation des réseaux d’habitat en archéologie: trois expériences », Mappemonde n83 (3-2006), en ligne sur http://mappemonde.mgm.fr

OUZOULIAS, P. 1991. « Eudes Rigaud et le vieux chemin Paris-Rouen », in : Cuisenier J. (dir.), 1991. Matière et figure. Paris, La Documentation française, Collection études et Travaux n³ : 17-42

PINCHEMEL P. et PINCHEMEL G., 1988. La face de la terre, éléments de géographie. Paris, Armand Colin, $515 \mathrm{p}$.

ROBERT, S. à paraître. " Des outils pour l'analyse de la transmission des trames et réseaux planimétriques : approches multiscalaires et traitements sur SIG », in : FAVORY, F., DELANNOY, J. J., GALOP, D. Colloque du réseau thématique pluridisciplinaire " Paysage et environnement, de la reconstitution du passé aux modèles prospectifs », Chilhac 27-30 sept. 2006, à paraître en 2008

ROBERT, S. 2007. "Modeling Ancient Roads ", in : Medieval Europe Paris 2007, 4e Congrès international d’Archéologie Médiévale, Institut National d’Histoire de l’Art, Paris, 3-8 septembre 2007. en ligne sur http://medieval-europe-paris-2007.univ-paris1.fr

ROBERT, S., 2003. L'analyse morphologique des paysages entre archéologie, urbanisme et aménagement du 
territoire. Exemples d'études de formes urbaines et rurales dans le Val-d'Oise. Thèse de doctorat. Paris, Université Paris I - Panthéon-Sorbonne, 621 p.

ROBERT, S. 2006a. «La résilience des réseaux routiers : l'exemple du Val-d’Oise », in : Bulletin AGER $n^{\circ} 15$, année 2005, Besançon, Presses Universitaires de Franche-Comté, 2006, pp. 8-14

ROBERT, S. 2006b. "Les itinéraires anciens traversant le département du Val-d'Oise », in : Bulletin archéologique du Vexin Français et du Val-d’Oise, n³8 : 7-23

Schnapp A. 1993. La conquête du passé. Aux origines de l’archéologie. Paris, éditions Carré, 511 p.

VAN LEUSEN, P. M. 2002. Pattern to process: methodological investigations into the formation and interpretation of spatial patterns in archaeological landscapes. Groningen, Rijkuniversiteit, 356 p.

VERHAGEN, P., GILI, S., MICO, R., RISCH, R. 1999. "Modelling Prehistoric Land Use Distribution in the Rio Aguas Valley (SE Spain)”, in : DINGWALL L. et al. (eds) : Archaeology in the Age of the Internet. Proceedings of the CAA97 conference, Oxford, BAR International Series, 750, CDRom

VION, E. 1989. «L'analyse archéologique des réseaux routiers : une rupture méthodologique, des réponses nouvelles », in : Paysages Découverts : 67-99.

ZAKŠEK, K., FOVET, E., NUNINGER, L., PODOBNIKAR, T. à paraître "Path modelling and settlement pattern”, in : Posluschny A., Lambers K., Herzog I. eds. - Layers of perception: Proceedings of the 35th Computer Applications and Quantitative Methods in Archaeology Conference, Berlin, Germany, April 2-6, 2007. Bonn (Germany): Habelt (Kolloquien zur Vor- und Frühgeschichte, vol. 10

\section{Illustrations :}

- - Grenier 1985 p. 347 Dès 934, A. Grenier attire l’attention sur la diversité des voies antiques dont certaines sont en structure légères et qu'il pense héritées de la période gauloise 